Int. J. Speleol. 10 (1978), pp. $323-330$

\title{
Sui Rhachomyces (Ascomycetes, Laboulbeniales) parassiti dei Duvalius italiani (Coleoptera, Carabidae, Trechini)
}

di

\section{W. ROSSI*}

\begin{abstract}
A study on Rhachomyces species (Ascomycetes, Laboulbeniales), parasitic on Italian Duvalius (Coleoptera, Carabidae, Trechini)
\end{abstract}

\section{SUMMARY}

The systematic position, variability and distribution of the two species of Rhachomleces parasitic on Italian Duvalius are defined on the basis of several new findings.

Rhachomyces stipitatus Thaxter was found in Liguria, central Italy, Sicily and Sardinia; its synonymy with R. capucinus Thaxter is confirmed and the ssp. pallictus Maire is considered of no systematic relevance.

R. maublancii, whose position is regularized by a Latin validating diagnosis, was only found on the Alps and previous records in other countries are questioned.

I dati riportati dalla letteratura sulle Laboulbeniali parassite dei Duvalius italiani sono pochi e si riferiscono per lo più al genere Laboulbenia (Fig. 13). Per quel che riguarda il genere Rhachomyces abbiamo praticamente due sole segnalazioni: quelle di R. maublanci, descritto su Duvalius baldensis e D. baldensis tombeanus (Lepesme, 1942), e quella più recente di Rhachomyces capucinus Thaxt., rinvenuto su Duvalius lepinesis (Balazuc, 1973). Ma Rhachomlces maublanci è un nomen nudum in base alle norme nomenclatoriali vigenti. non essendo la descrizione originale accompagnata da una diagnosi latina, mentre $R$. capucinus è stato recentemente messo in sinonimia con $R$. stipitatus Thaxt. (Rossi, 1978). Lo scopo di questo lavoro, dunque, sarà quello di definire la posizione sistematica delle specie di Rhachombees parassite dei I Ju'alius italiani e di supplire alla sorprendente mancanza di informasioni sull'argomento.

* Via P. Trogo 28, (0)136 Romia, Italia 
Rhachomyces stipitatus Thaxter 1900 (Figg. 1-7)

Abbiamo rinvenuto questo parassita sui seguenti insetti:

- Duvalius bensai (Gestro): grotta di M. Cucco, 17 U (Perugia).

- D. bensai lombardi Straneo: grotte di Genga, presso Fabriano (Ancona).

- D. canevai apenninus (Gestro): Tana Rimilegni, $163 \mathrm{LI}$, presso Bardineto e tana del Pecetto, $569 \mathrm{LI}$, presso Magliolo (Savona).

- D. cerrutii Sbord. e Di Dom.: pozzo l'Arcaro, 340 LA, sul M. Siserno (Frosinone).

- D. lepinensis Cerruti: Ouso del Pozzo Comune, 274 LA, e Ouso dell'Isola, 24 LA, presso Carpineto Romano (Roma); grotta di Fonte Serena, 424 LA, e pozzo del Faggeto, 343 LA, presso Supino (Frosinone).

- D. sardous (Dodero) : grotta Conca 'e crapa, $30 \mathrm{SA}$, M. Turuddò presso Lula (Nuoro).

- D. siculus (Baudi): bosco della Ficuzza (Palermo).

Lo stesso parassita è anche presente su due specie inedite di Duvalius, provenienti l'una dai Monti Lepini e l'altra dagli Aurunci.

Da quanto sopra si ricava che appartengono a Rhachomyces stipitatus sia l'esemplare segnalato da Franciscolo su Duvalius canevai apenninus (1955, nota in fondo alla pagina 65), che quelli indicati da Sbordoni e Di Domenico su D. cerrutii (1967, pag. 177).

I. 'abbondanza del materiale esaminato permette di chiarire alcune questioni di carattere morfologico che, almeno in parte, sembrano responsabili della confusione che è stata fatta intorno a questa specie. Va subito detto che il fungo risente della posizione che occupa sull'insetto ospite: questo fatto, riscontrabile in altre Laboulbeniali, sembra essere sfuggito a quanti si sono occupati della sistematica di questo genere. Negli esemplari impiantati sulle elitre ed il protorace, il peduncolo del peritecio è di lunghezza molto variabile: da quasi nullo fïno ad una volta e mezzo l'altezza del peritecio (Figg. 1-2); negli esemplari che si trovano sull'addome, il peritecio è più spesso sessile o quasi. Nel primo caso l'asse principale del fungo è formato, per quel che riguarda i parassiti italiani, da 6-10 cellule subquadrate o un po' più alte che larghe; nel secondo, le cellule dell'asse sono schiacciate, sono in numero maggiore (fino a 15), e ciascuna di esse dà origine ad un numero di cellule appendicolate più al10 rispetto a quanto si osserva nel primo caso. I funghi che crescono sui femori c le tibic sono simili a quelli che crescono sull'addome per quel che riguarda i caratteri del ricettacolo, ma non è raro osservare sui femori parassiti con il peritecio peduncolato; sui tarsi si trovano invece esemplari con if ricettacolo estremamente ridotto. Si osservino in proposito le foto n. 3 e 4 : i due funghi cono stati rinvenuti su di uno stesso insetto ospite, l'uno sulle elitre (Fig. 4) e l'altro sul femore posteriore destro, in prossimità dell'articolazione con la tihia (Fïg. 3). 

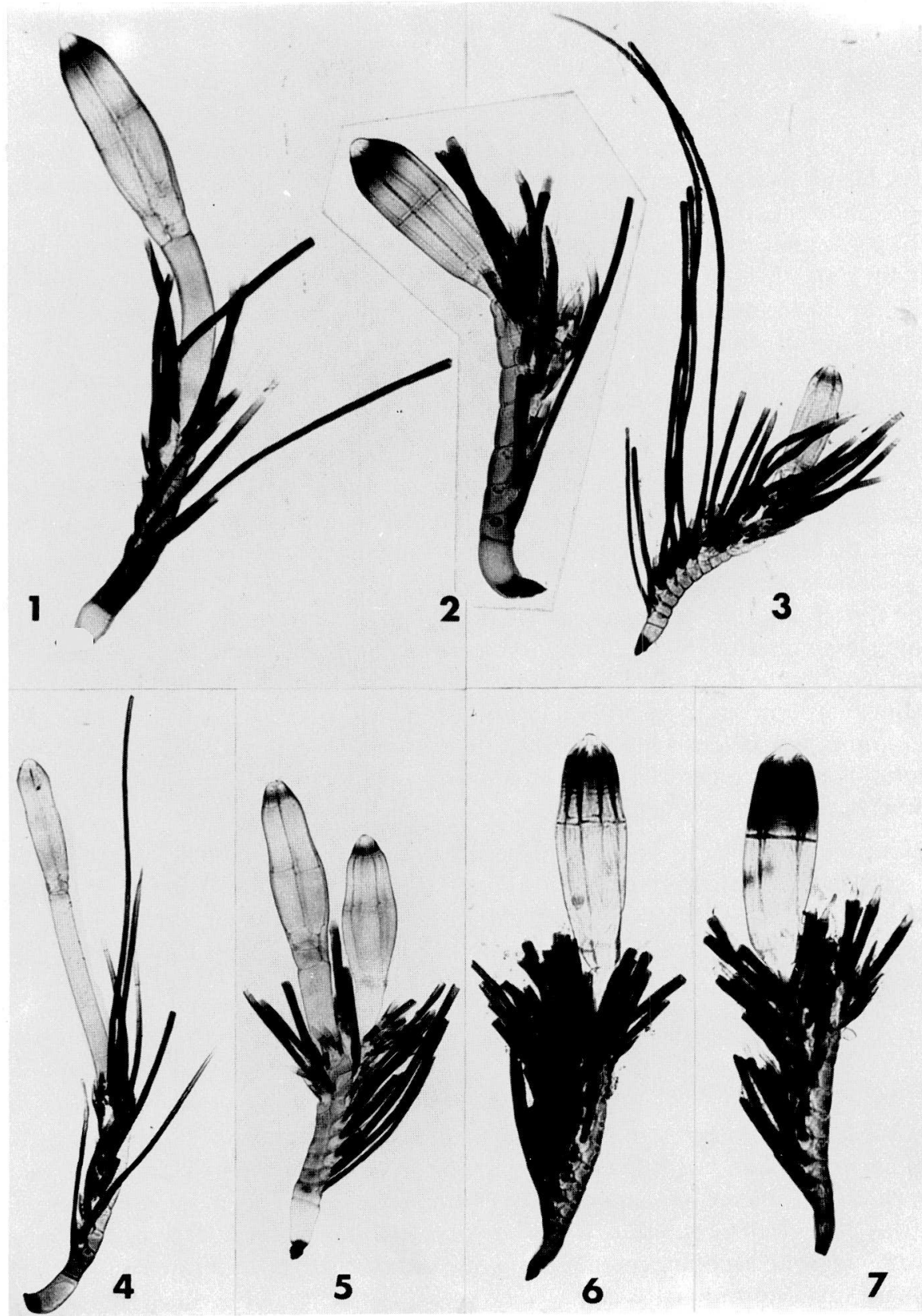

Figg. 1-7: Rhachomyces stipitatus Thaxter.

Figg. 1 e 2. Individui rinvenuti sulle elitre di un Duvalius lepinesis $(1: \times 180,2: \times 225)$.

Figg. 3 e 4 . Due parassiti di $D$. sp. dei M. Lepini; 3 . Individuo rinvenuto su di un femore $(\times 130)$; 4. Individuo rinvenuto sulle elitre dello stesso insetto $(\times 140)$.

Fig. 5. Individuo rinvenuto sulle elitre di $D$. bensai $(\times 150)$.

Figg. 6 e 7 . Individui rinvenuti su di un femore di D. sardous $(6: \times 160,7: \times 185)$. 
Altro carattere piuttosto variabile è costituito dalla pigmentazione del peritecio. Generalmente in questa specie si osserva un anello bruno più o meno scuro e più o meno netto in posizione preapicale (Figg. 1, 2, 3, 5); in qualche caso, però, questo anello è appena visibile, come in Fig. 4. Similmente ai caratteri già trattati, tali variazioni possono essere osservate tra esemplari viventi su di uno stesso insetto: anche questo a proposito risulta particolarmente significativo il confronto tra le Figg. 3 e 4 . Un discorso a parte va fatto per i parassiti di Duvalius sardous, nei quali la zona pigmentata è molto più scura e più estesa rispetto a tutti gli altri esemplari italiani (Figg. 6-7).

In base a quanto sopra esposto sulla variabilità di questo fungo, non possiamo che confermare la sinonimia tra Rhachomyces stipitatus Thaxter 1900 e $R$. c $a$ pucinus Thaxter 1932: le due specie sono state infatti separate in base a presunte differenze nell'intensità della pigmentazione del peritecio, nella lunghezza del peduncolo dello stesso e nel numero delle appendici. Abbiamo visto come questi caratteri possono variare anche tra esemplari che si trovano su di uno stesso insetto e come gli ultimi due possono essere condizionati dalla posizione occupata dai funghi sull'ospite. La variabilità osservata nella pigmentazione del peritecio, poi, priva di qualsiasi valore sistematico la sottospecie $R$. stipitatus ssp. pallidus Maire (1912), descritta su Duvalius iblis (Peyer.) di Algeria, il cui unico carattere differenziale rispetto al tipo sembra quello di avere il peritecio meno pigmentato.

Riportiamo, infine, le dimensioni rilevate sugli esemplari italiani. Altezza dalla punta del piede all'apice del peritecio: $235-615 \mu$ Peritecio: $120-195 \times 35-80 \mu$. Lunghezza massima del peduncolo del peritecio: $235 \mu$ Lunghezza massima delle appendici (rilevata su esemplari rinvenuti sul metasterno di un Duvalius appartenente alla nuova specie dei Monti Lepini): $1200 \mu$.

\section{Rhachomyces maublanci Lepeseme 1942, nomem nudum}

Come abbiamo accennato precedentemente, questo parassita è stato descritto su Duvalius baldensis Putz. e sulla sua sottospecie tombeamus (ianglb. I:" stato successivamente segnalato su $D$. roubali Jaenn. di Romania (Banhegyi, 1949) e su Geotrechus saulcyi ssp. demauxi Coiff. (Boyer-I efevre, 1966); le fotografie che accompagnano queste due ultime segnalazioni non sono molto chiare, ma sembrano riferirsi ad altre specie: la forma del peritecio del fungo in questione, infatti, è cosi caratteristica da lasciare pochi dubbi in proposito.

Noi abbiamo osservato Rhachomyces maublanci su:

- Duvalius baldensis baldensis Putz: M. Baldo, Cima Valdritta e (ima Telegrafo, (Verona).

- D. haldensis cartolarii Pom.: M. I essini, cima Sparavieri (Verona). 
- D. baldensis pasubianus Putź.: M. Lessini, Passo Pelagatta (Vicenza).

- D. breiti montisgrappae Agazzi: M. Grappa, presso la cima (Treviso).

Anche in questa specie abbiamo potuto rilevare alcune variazioni morfologiche collegate alla posizione del fungo sull'ospite. Come nel caso precedente, le cellule del ricettacolo sono più numerose, più schiacciate e munite di un numero maggiore di cellule appendicolate negli esemplari impiantati sull'addome, che raggiungono anche le dimensioni maggiori (Fig. 10); per contro, il peritecio è sempre sessile.

Attribuiamo a $R$. maublanci anche gli esemplari rinvenuti su:

- Duvalius adamellensis Jeann.: Passo delle Portole, Lavenone (Brescia).

- D. boldorii vaghezzae Ghid.: Pian di Vaghezza, Tavernole sul Mella (Brescia).

- D. winklerianus brescianus Jeann.: Val Trompia e Val Porcino, Lumezzane; Büs de le Bedole, 221 LO (Brescia).

- D. winklerianus brescianus Jeann.: presso Pezzoro e presso Tavernole sul Mella (Brescia).

I parassiti di questo secondo gruppo di insetti sono sostanzialmente simili a quelli degli insetti citati precedentemente: solo il peritecio può presentarsi leggermente peduncolato (Fig. 11).

Attribuiamo alla stessa specie, infine, anche i Rhachomyces rinvenuti su:

- Iuvalius gentilei (Giestro): grotta delle Fascette, 132 PI, presso Viozene (Cune()).

- I). gentilei ingaunus (Dodero): grotta della Sierra, 279 PI, presso Caprauna (cuneo).

- I). spagnoloi (Ciestro): Abisso di M. Pietravecchia, 628 PI; grotta del Latte di Monte (non catastata) presso Melosa; grotta E.4, 631 LI, presso Pigna; Tana Bertrand, 104 I.I, presso Badalucco (Imperia).

I a presenza di un Rhachomyces non identificato su D. spagnoli dell'ultima delle località citate è stata già segnalata da Balazuc (1970).

I funghi osservati sulle parti superiori di questi ultimi ospiti sono mediamente più grandi ed hanno il peritecio nettamente peduncolato (peducolo lungo fino a 15()$\mu)$ (Fig. 12); anche in questo caso, però gli esemplari impiantati sull'addome é sulle /ampe degli insetti hanno il peritecio assolutamente sessile: non riteniamo percio di poter separare questi parassiti da $R$. maublanci.

Non rimanc, a questo punto, che regolarizzare la posizione di questa specie dotandola di una diagnosi latina e completarne la descrizione in base alle osservarioni personali. 

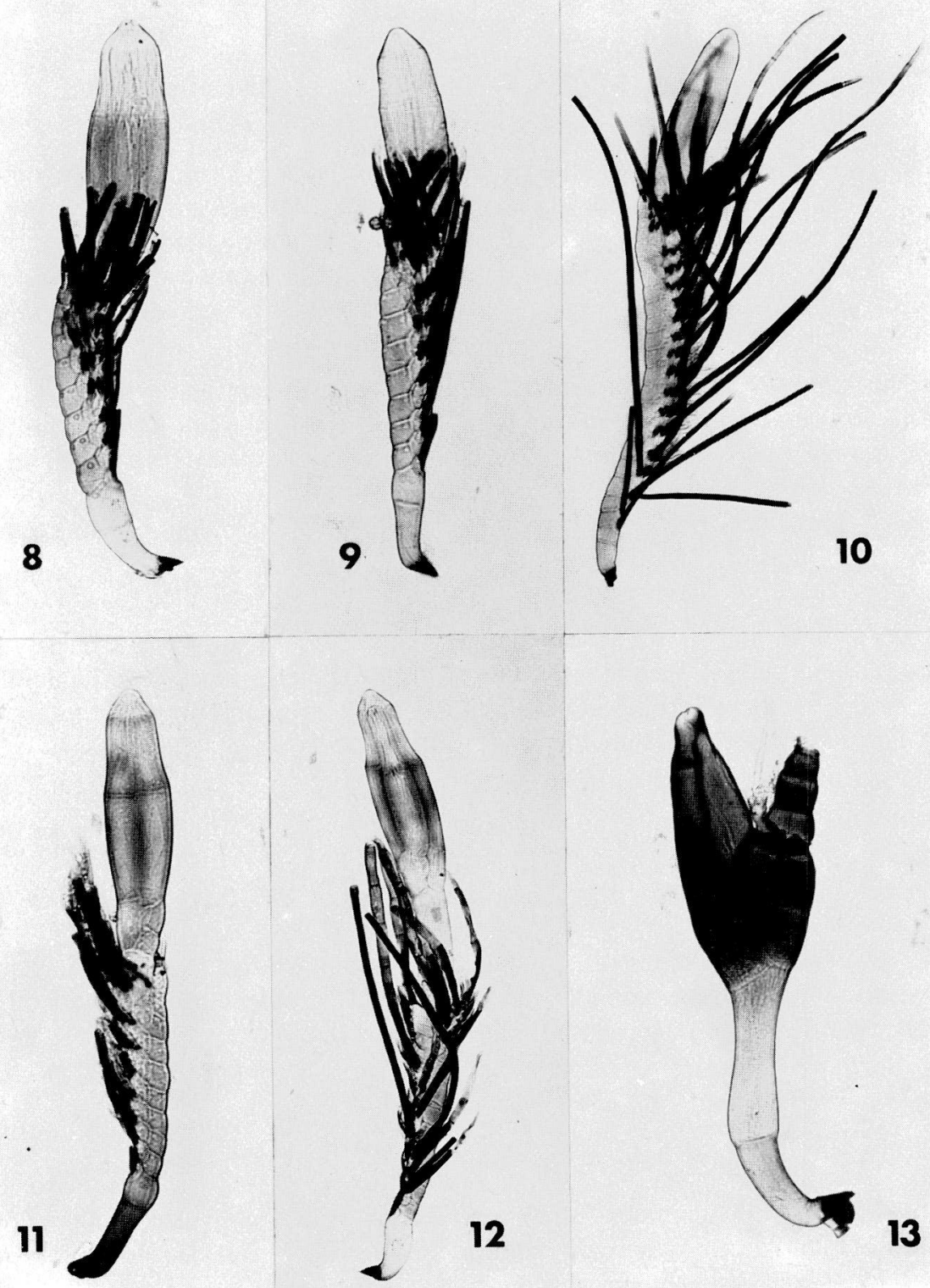

Figg. 8-12. Rhachomyces maublancii Lepesme ex W. Rossi.

Figg. 8 e 9 . Individui rinvenuti sulle elitre di Duvalius baldensis $(8: \times 190 ; 9: \times 200)$.

Fig. 10. Individuo rinvenuto sull'addome di $D$. winklerianus aequalius $(\times 135)$.

Fig. 11. Individuo rinvenuto sulle elitre di $D$. boldorii vaghezzae $(\times 185)$.

Fig. 12. Individuo rinvenuto sulle elitre di D. spagnoloi $(\times 150)$.

Fig. 13. Laboulbenia vulgaris Peyritsch: individuo rinvenuto sull'addome di Duvalius doderoi $(\times 190)$. 


\section{Rhachomyces maublancii Lepesme ex W. Rossi}

Praecipuus axis rectus vel parum arcuatus, ex 8-12 sucinis quasi quadriangulis cellulis constans, gradatim maiores usque ad perithecii insertionem, sed supra hanc multo minores. In speciminibus supra hospitis abdomen receptaculi cellulae plures (usque ad 20) et compressae sunt. Stigmata parum conspicua. Cellulae appendiculatae magnae, eodem colore quam cellulae praecipui axis. Antheridia decolorata, elongata, collo parum flexo. Appendices tenues, in maturis speciminibus apicem perithecii plerumque non superantes, sed in immaturis interdum longissimae. Perithecium aut sessile aut pediculo instructum, sucinum vel cinerascens in inferiori parte, in superiori vero hyalinum; post medium manifesto contractum, ad apicem deinde in conum extenatum, rotundato apice.

Ricettacolo rettilineo o leggermente arcuato, formato da 8-12 cellule ambrate, subquadrate, di dimensioni leggermente crescenti fino all'inserzione del peritecio ma bruscamente più piccole al di sopra di essa. Negli esemplari situati sull'addome dell'ospite le cellule del ricettacolo sono più numerose (fino a 20), più chiare e più schiacciate. Stimmi non molto evidenti. Cellule appendicolate grandi, concolori con quelle del ricettacolo. Anteridi chiari, allungati, con il collo leggermente incurvato. Appendici esili, quasi mai sorpassanti l'apice del peritecio negli esemplari maturi, ma talvolta lunghissime in quelli immaturi. Un solo peritecio (rarissimamente due) sessile o peduncolato, ambrato o grigio pellucido nella parte inferiore, ialino in quella apicale; la sua larghema comincia a decrescere regolarmente poco al di sopra della metà della sua alte» e si restringe bruscamente in una porzione terminale conica, ad apice arrotondato ed indifferenziato.

Altezza dalla punta del piede all'apice del peritecio: $285-595 \mu$. Peritecio: 13()$190 \times 50-75 \mu$. Lunghezza massima del peduncolo del peritecio (osservata in un esemplare parassita di $D$. spagnoloi): $150 \mu$. Lunghezra massima delle appendici (osservata in un esemplare immaturo rinvenuto sull'addome di un $I$ ). spagnoloi): $855 \mu$.

Le due specie di Rhachomyces parassite dei Duvalius italiani sono facilmente distinguibili tra di loro anche se sono, ovviamente, affini. Il carattere differenziale più vistoso è fornito dall'anello scuro preapicale del peritecio di $R$. stipitatus, che in genere permette, da solo, di distinguere a prima vista i duc parassiti. Inoltre il peritecio è sempre molto chiaro, di colorarione gialla o grigiastra in $R$. maublancii, mentre è brunastra in $R$. stipitatus; il ricettacolo i giallo chiaro con stimmi poco visibili nella prima specie, mentre in $R$. stipitutus questo è bruno, a volte molto scuro, formato da cellule generalmente piu allungate, con stimmi ben visibili. (ili anteridi di $R$. mauhlancii sono piu piccoli, piu sottili, più chiari, e le appendici più esili, meno piementate, formate da cellu- 
le più brevi; le stesse appendici a maturità non superano mai in altezza l'apice del peritecio in $R$. maublancii (la foto n. 10 raffigura un esemplare non ancora maturo), mentre sono scure, rigide, a volte lunghissime anche a maturità in $R$. stipitatus.

E' interessante notare che le due specie hanno areali differenti: $R$. stipitatus ha una distribuzione perimediterranea ed in Italia è presente nella penisola e nelle isole maggiori, mentre $R$. maublancii è presente solo lungo la catena alpina.

Tra quanti hanno fornito del materiale per il presente articolo, desideriamo ricordare J. Balazuc, I. Bucciarelli, R. Poggi, V. Sbordoni ed A. Vigna Taglianti per l'abbondanza del materiale messo a nostra disposizione insieme a suggerimenti e notizie. A loro, ed a tutti gli altri che sarebbe lungo menzionare, indirizziamo i nostri più sentiti ringraziamenti. Ringraziamo, inoltre, L. Zenobi e G. Biagiotti per la collaborazione alla tavola fotografica e L. Cajani per la traduzione della diagnosi in latino.

\section{RIASSUNTO}

In base a numerosi nuovi reperti viene definita la posizione sistematica, la variabilità e la distribuzione delle due specie di Rhachomyces parassite dei Duvalius italiani.

Rhachomyces stipitatus Thaxter è stato rinvenuto in Liguria, Italia centrale, Sicilia e Sardegna: ne viene confermata la sinonimia con $R$. capucinus Thaxter e viene considerata priva di valore la ssp. pallidus Maire.

$R$. maublancii Lepesme, la cui posizione sistematica viene regolarizzata dando per essa una diagnosi latina, è invece segnalato solo lungo la catena alpina.

\section{BIBLIOGRAFIA}

BANHEGYI J., 1949 - Les Laboulbéniales de la Transylvanie. Index horti bot. Univ. budap., 7 : 93-101.

BALAZUC J., 1970 - Commentaire sur les Rhachomyces parasites des Trechidae, avec descriptions de formes nouvelles. Ann. Soc. entomol. Fr., 6 (3): 677-699.

BALAZUC J., 1973 - Laboulbéniales de France. Bull. Soc. linn. Lyon, 42 (9) : 244-256.

BOYER-LEFEVRE N. H., 1966 - Les Laboulbéniales des Trechinae cavernicoles pyrénéens. Ann. Spéleol., 21 (3) : 775-794.

FRANCISCOLO M. E., 1955 - Fauna cavernicola del Savonese. Ann. Mus. Civ. Stor. Nat. Genova "Giacomo Doria», LXVII : 1-223.

LEPESME P., 1942 - Révision des Rhachomyces paleartiques (Laboulbeniaceae). Bull. Soc. myc. Fr., $58(1-2): 57-80$.

MÁIRE R., 1912 - Contribution à l'étude des Laboulbéniales de l'Afrique du Nord. Bull. Soc. Hist. Nat. Afr. Nord. 4 (9) : 194-199.

ROSSI W., 1978 - Sulle Laboulbeniali (Ascomycetes) parassite dei Trechini di Turchia (Coleoptera, Carabidae). In: Fauna Ipogea della Turchia. Quaderni di Speleologia, 3:1-8.

SBORDONI V. e DI DOMENICO N., 1967 - Una nuova specie cavernicola di Duvalius dell'Appennino centrale. Fragm. Entomol., V (2) : 165-180.

THAXTER R., 1900 - Preliminary diagnoses of new species of Laboulbeniaceae. Proc. Amer. Acad. Arts \& Sci., 35 (9): 151-209.

THAXTER R., 1931 - Contribution towards a monograph of the Laboulbeniaceae. Part. V. Mem. Amer. Acad. Arts \& Sci., 16 (1) : 1-432. 